\title{
Preliminary Development of an Automated Optical Inspection System for Quality Control of Circular Saw Inserts
} auSMT:

\author{
Wen-Tung Chang1, *, Shui-Fa Chuang ${ }^{2}$, Cheng-Yu Li ${ }^{3}$, \\ Fang-Jung Shiou ${ }^{3}$, and Geo-Ry Tang ${ }^{3}$ \\ ${ }^{1}$ Department of Mechanical and Mechatronic Engineering, National Taiwan Ocean University, Taiwan \\ ${ }^{2}$ Opto-Mechatronics Technology Center, National Taiwan University of Science and Technology, Taiwan \\ ${ }^{3}$ Department of Mechanical Engineering, National Taiwan University of Science and Technology, Taiwan \\ (Received 4 October 2012; Accepted 22 October 2012; Published on line 1 March 2013) \\ *Corresponding author: wtchang@mail.ntou.edu.tw; wentungchang@gmail.com \\ DOI: 10.5875 /ausmt.v3i1.159
}

Abstract: Circular saw inserts are fundamental and high-value-added cutting tool inserts used for sawing, slot milling, and other related machining processes. In their mass production, sintered blanks are first made by powder metallurgy and then precisely form-ground to generate the geometric shapes and dimensions required for cutting action. In order to ensure quality in the production of circular saw inserts, the defects of sintered blanks and geometric accuracy of semi-products must be inspected before and after the form-grinding process, respectively. To this end, this paper presents the preliminary development of an automated optical inspection (AOI) system for quality control of circular saw inserts. A prototype system was designed and constructed in this study. Image processing procedures were then developed to perform the calibration process of the prototype system, defect detection of the sintered blanks, and geometric measurement of the form-ground semi-products. Experiments were conducted to inspect insert samples and test the feasibility of the developed prototype system. Preliminary experimental results verified the presented prototype system and image processing procedures as being able to inspect certain circular saw inserts for ensuring quality control.

Keywords: Automated optical inspection (AOI); Circular saw insert; Quality control; Defect detection; Geometric measurement

\section{Introduction}

Circular saw inserts are fundamental and highvalue-added cutting tool inserts used for sawing, slot milling, and other related machining processes. In practice, a number of cutting tool inserts must be assembled around the circumference of a circular saw plate to form a functional circular saw blade [1, 2]. Figure 1 shows the appearance and application of the Yih Troun circular saw blade with cutting inserts [3]; a schematic diagram of the insert is shown in Figure 2. As can be seen in Figure 1(a)-(b), the inserts are firmly clamped by the circular saw plate through their upper and lower V-shaped grooves, which engage with a specially designed jaw-shaped structure of each tooth of the plate. The inserts are usually made of tungsten carbide alloy, and the circular saw plate of heat-treated tool steel. In mass production of the inserts, sintered blanks are first made by powder metallurgy (PM) and then precisely form-ground to generate the required geometric shapes and dimensions for cutting action. In order to ensure 
quality in the production of circular saw inserts, the defects of sintered blanks and geometric accuracy of semi-products must be inspected before and after the form-grinding process, respectively. To this end, this paper presents the preliminary development of an automated optical inspection (AOI) system for quality control of Yih Troun circular saw inserts.

\section{Setup of the Inspection System}

A prototype inspection system was designed and constructed to perform the inspection of the circular saw inserts. Figure 3 shows the final setup of the prototype system. The inspection system mainly consisted of a rotary gripper module and a machine vision module. The

Wen-Tung Chang was born in 1978 in Taipei, Taiwan. He received his Ph.D. Degree in Power Mechanical Engineering from the National Tsing Hua University in 2007 for his work on mechanical error analysis of planar cam mechanisms and its applications. His Ph.D. dissertation won him an Outstanding Ph.D. Dissertation Award from the Chinese Society of Mechanism and Machine Theory in November 2007. In January 2008, Dr. Chang was recruited as a Defense Industry Reserve Duty Officer of Opto-Mechatronics Technology Center at the National Taiwan University of Science and Technology in Taipei, Taiwan, where he served as a Post-doctoral Researcher for the remaining four years of his military service. He led several projects to innovate and develop automated optical inspection systems for measurement and quality control of precision cutting tool products. In February 2012, Dr. Chang joined the National Taiwan Ocean University in Keelung, Taiwan, where he is currently an Assistant Professor in the Department of Mechanical and Mechatronic Engineering. His current teaching and research interests include kinematics and dynamics of machinery, mechanism and machine design, manufacturing processes and devices, precision mechanical measurement, and automated optical inspection.

Shui-Fa Chuang was born in 1977 in Pingtung, Taiwan. He received his Ph.D. Degree in Mechanical Engineering from the National Taiwan University of Science and Technology in 2010 for his work on developing optical inspection systems for measurement of microdrills. In October 2010, Dr. Chuang was recruited as an R\&D Alternative Military Service Draftee of Opto-Mechatronics Technology Center at the National Taiwan University of Science and Technology in Taipei, Taiwan, where he is currently a Postdoctoral Re-searcher. His current research interests include automated optical inspection, mechatronic technology, and LabVIEW-based programming.

Cheng-Yu Li was born in 1985 in Nantou, Taiwan. He received his Master's Degree in Mechanical Engineering from the National Taiwan University of Science and Technology in 2011 with work on developing an optical inspection system for quality control of circular saw inserts. In October 2011, Mr. Li was recruited as an R\&D Alternative Military Service Draftee at Hiwin Technologies Corporation in Taichung, Taiwan. He is currently an R\&D Engineer.

Fang-Jung Shiou received his Ph.D. Degree from Rheinisch-Westfälische Technische Hochschule in Aa-chen, Germany. He is currently a Professor and Chair of the Department of Mechanical Engineering, as well as Director of the Opto-Mechatronics Technology Center at the National Taiwan University of Science and Technology in Taipei, Taiwan. His current teaching and research interests include engineering metrology, precision manufacturing, and opto-mechatronic technology.

Geo-Ry Tang received his Ph.D. Degree from Texas A\&M University in the USA. He is currently a Professor in the Department of Mechanical Engineering at the National Taiwan University of Science and Technology in Taipei, Taiwan. His current teaching and research interests include tolerance analysis and design of mechanical assemblies, computer-aided design, and automated optical inspection. rotary gripper module essentially consisted of a rotary gripper subassembly coupled with an Oriental Motor/Vexta PK566NAW five-phase stepping motor. As shown in Figure 3(b), the fundamental component of the rotary gripper subassembly was a precision main shaft, which was driven by the stepping motor. The radial and axial runout amounts of the main shaft were both verified to be less than $1 \mu \mathrm{m}$ by a Mitutoyo ID-C112CMXB 543-395B digimatic indicator. A lower member and a pair of miniature linear guides were mounted to the end face of the main shaft. An upper member was mounted to the sliders of the miniature linear guides, so that it could translate along the radial direction of the main shaft, while the lower member remained stationary with respect to the main shaft. A pair of tension springs was used to provide gripping force between the upper and lower members. Thus, the upper and lower members formed a simple gripper that could be manipulated manually. As shown in Figure 3(c), the lower member was characterized by a front and a back datum. Both datums were precisely ground to ensure that they were parallel to each other as accurately as possible. The perpendicular distance between the front and back datums (denoted by $L_{0}$ ) was measured to be $9.745 \mathrm{~mm}$ by a Mitutoyo Crysta-Apex C544 coordinate measuring machine. In addition, the upper and lower members were designed to have $\mathrm{V}$-shaped contact tips for engaging with the $V$-shaped grooves of the insert under inspection; this allowed the insert to be firmly held during rotation, which is required in the inspection process. The machine vision module mainly consisted of an Aisys Altair U500 complementary metal-oxidesemiconductor (CMOS) camera coupled with a Moritex MML1-ST65D telecentric lens and a Moritex MCBPCW3430 backlight illuminator. The optical axis of the telecentric lens was set perpendicular to the axial direction of the main shaft as accurately as possible, while the location of the CMOS camera could be finely adjusted along three orthogonal directions for positioning and focusing purposes. As a result, the rotary gripper module was used to hold and rotate the sintered blanks or form-ground semi-products of the inserts under inspection, and the machine vision module to capture images of the inserts at specified orientations.

The prototype inspection system was controlled by a host personal computer (PC). A National Instruments (NI) $\mathrm{PCl}-7340$ motion control card was installed in the host PC to manipulate the stepping motor. The stepping motor was driven through an Oriental Motor/Vexta CRD5114P stepping motor driver, and was set to an angular resolution of $0.0036^{\circ} /$ step. The CMOS camera was connected to the host PC using a universal serial bus (USB) port. Each captured digital image consisted of a 
$2592 \times 1944$ array of pixels with grayscale intensity values ranging from 0 to 255 . To operate the prototype system through the host $P C$, the required human-machine interface software and motion control and image processing programs were developed and integrated in the NI LabView environment.

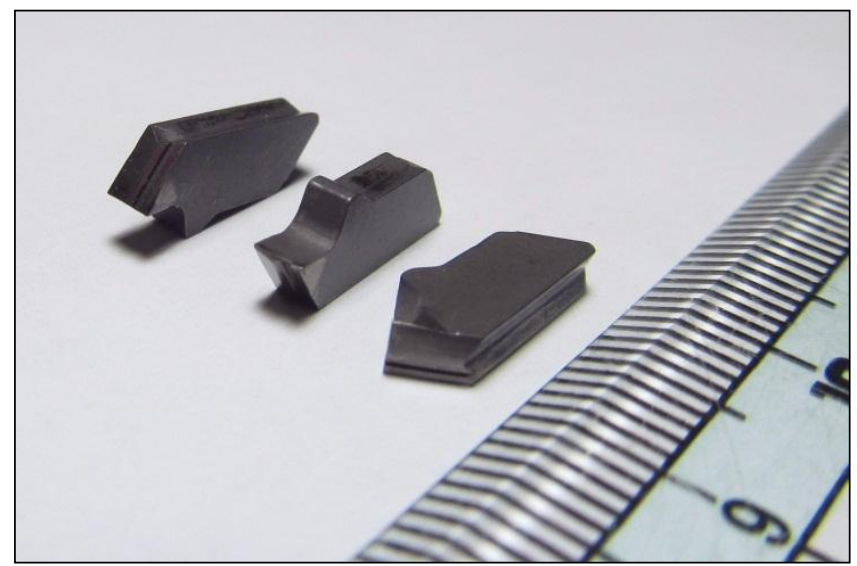

(a)

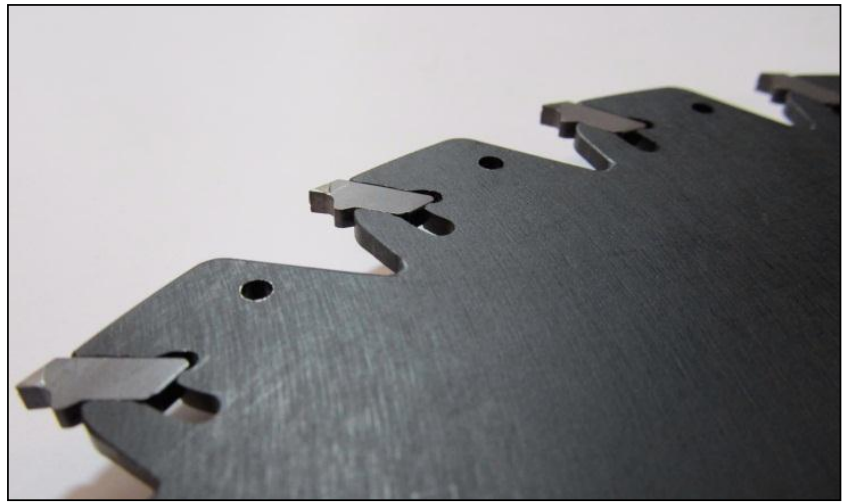

(b)

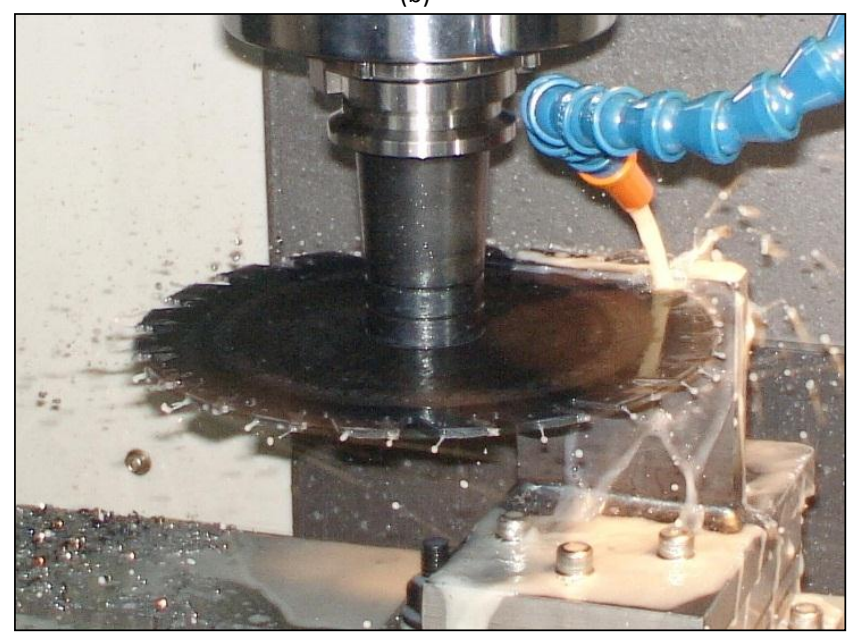

(c)

Figure 1. The appearance and application of the Yih Troun circular saw blade with cutting inserts [3]: (a) the finished inserts, (b) a local view of the inserts assembled to a circular saw plate, and (c) the circular saw blade in use, sawing a rectangular workpiece into slices.

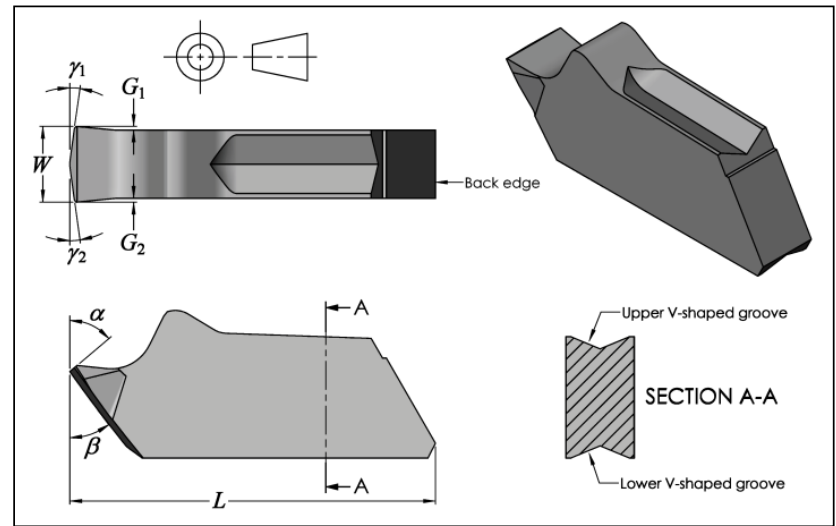

Figure 2. Schematic diagram of a circular saw insert.
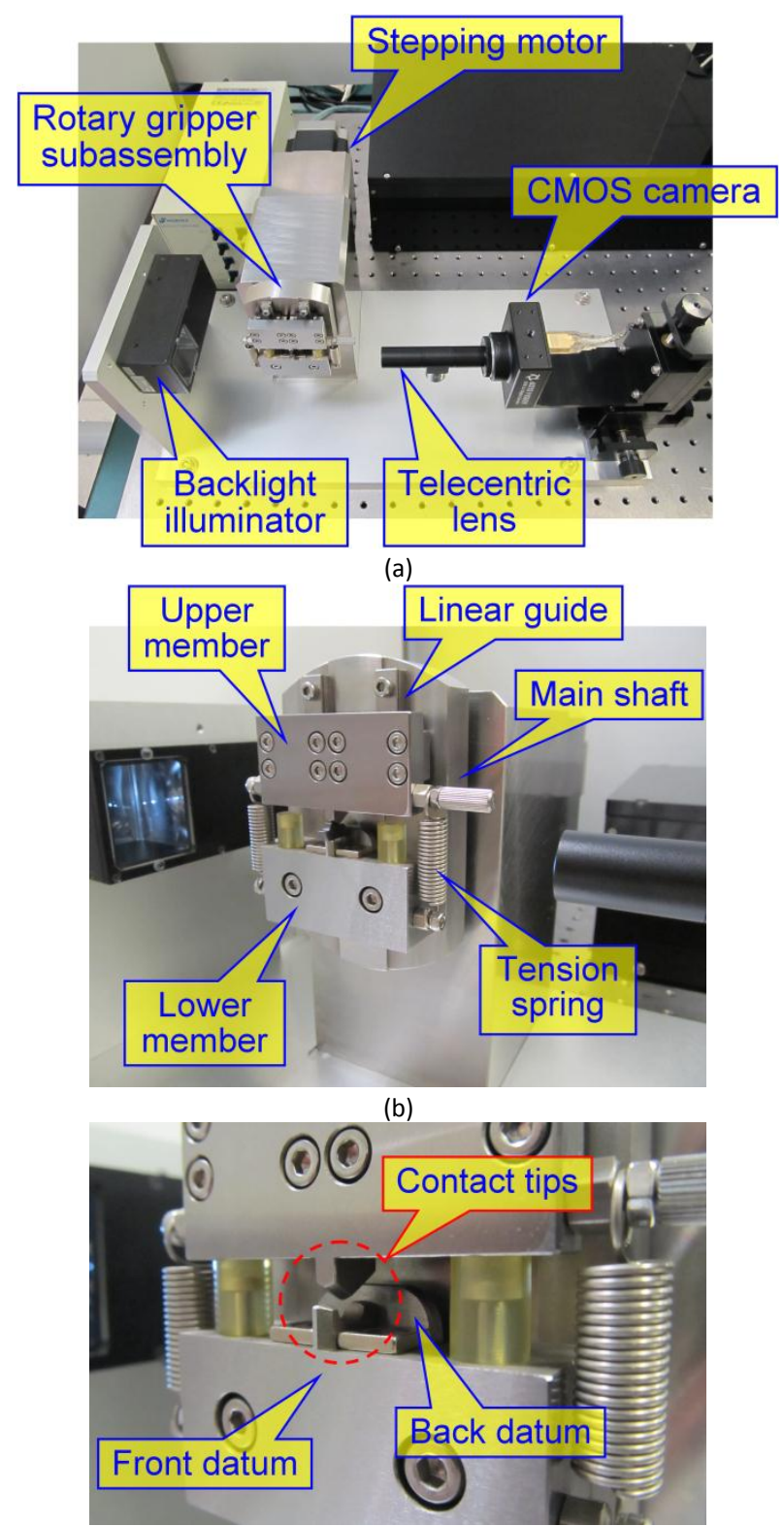

(c)

Figure 3. The constructed prototype system: (a) setup of the entire system, (b) rotary gripper subassembly details, and (c) characteristics of the gripper. 


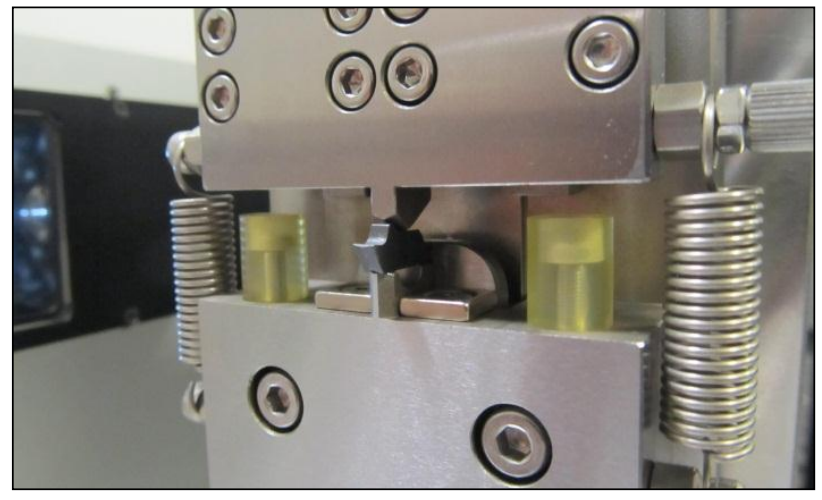

(a)

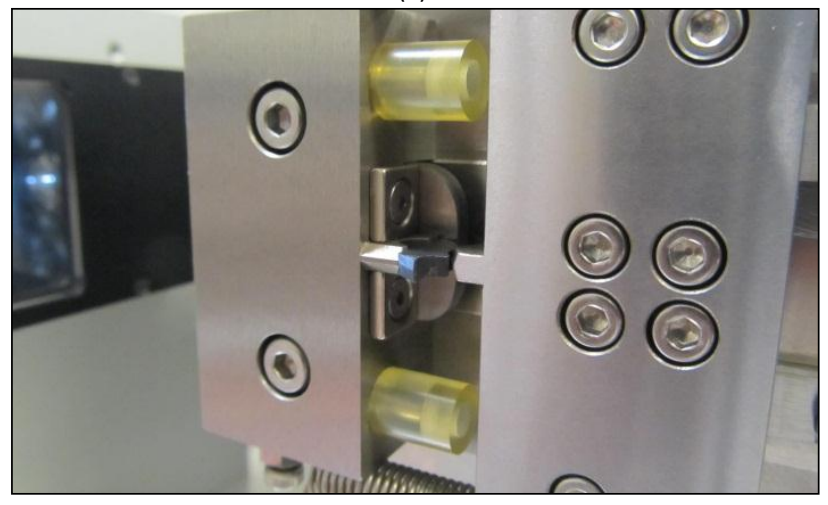

(b)

Figure 4. The inspection of an insert: (a) setup of the insert for capturing side images and (b) setup of the insert for capturing face images.

During inspection, the insert was placed between the contact tips of the upper and lower members of the gripper, with the back edge of the insert (as labeled in Figure 2) in contact with the back datum of the lower member. Images of the insert were captured by the machine vision module, and the insert was rotated and positioned by the rotary gripper module. After performing fine adjustment of the CMOS camera so that the characteristics of the insert could appear in its field of view (FOV), parallel light emitted from the backlight illuminator was projected on the insert, creating a silhouette before the telecentric lens, which was sensed and captured by the camera as a digital image. As shown in Figure 4(a), an insert under inspection was rotated to a specified angular position for side image capture. Figure 4 (b) then shows its face image capture configuration (the insert was rotated $90^{\circ}$ clockwise from the side image position). The captured side and face images are used to detect defects in or to conduct geometric measurements of the insert by the image processing procedures described in the following section.

\section{Image Processing Procedures}

The image processing procedures used for the calibration process of the prototype AOI system, the defect detection of the sintered blanks, and the geometric measurements of the form-ground semiproducts, were developed and are introduced in this section.

Calibration Process

The calibration process aims to determine the conversion factor of the machine vision module as well as the angular phase of the rotary gripper module. Both tasks could be simultaneously undertaken with the specially designed calibration gage shown in Figure 5(a); the gage consisted of a Renishaw A-5003-1325 stylus assembled into the central bore of a miniature fixture block with a locational interference fit. The ruby ball of the stylus has a nominal diameter of $1 \mathrm{~mm}$ and a DIN-5401 ball grade of G5. The fixture block was designed to have $V$-shaped grooves for engaging with the V-shaped contact tips of the gripper, in order for it to be firmly held during calibration, as shown in Figure 5(b). The central axis of the stylus was set with a small eccentricity to the rotational axis of the main shaft. Rotation of the stylus with a constant angular velocity produced a simple harmonic motion of the ruby ball, which was observable through the machine vision module.
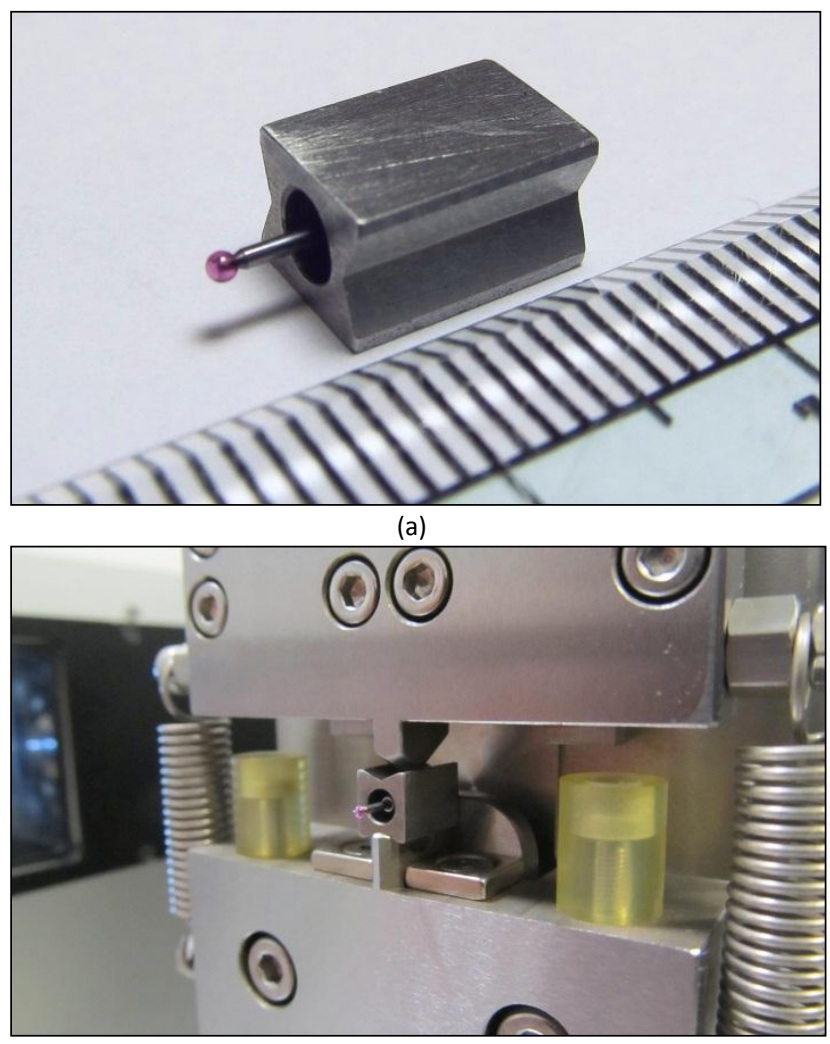

(b)

Figure 5. Calibration of the prototype system: (a) the specially designed calibration gage and (b) setup of the calibration gage for the calibration process. 


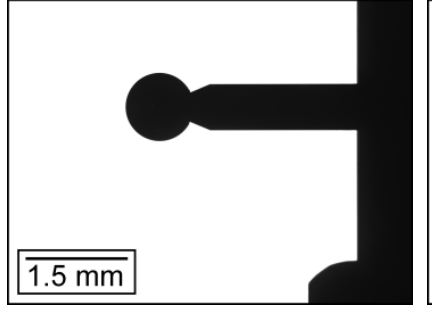

(a)

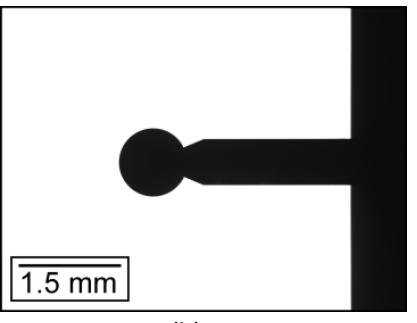

(b)

Figure 6. Captured images of the stylus when located at (a) its highest and (b) its lowest position.

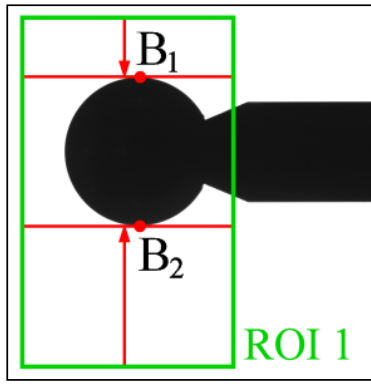

(a)

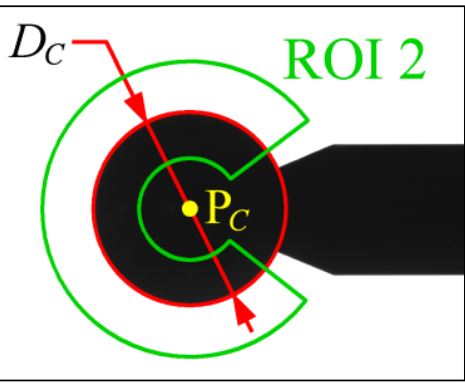

(b)

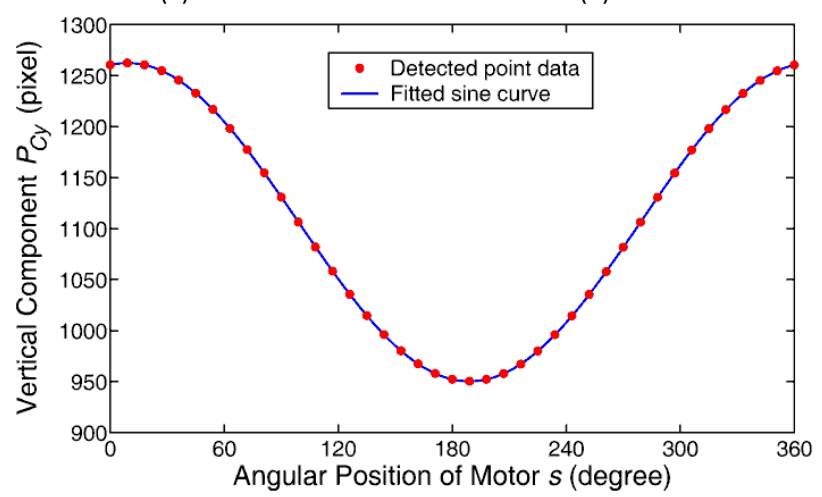

(c)

Figure 7. An illustrated example of the calibration process: (a) the use of the clamp function [4], (b) the use of the spoke function [4], and (c) a representative angular phase calibration result.

For calibration, the stylus (initially at an arbitrary angular position) was rotated intermittently with an angular interval of $9^{\circ}$ for one revolution, and its images were captured when it dwelled after each rotation interval. Figure 6 shows two captured images of the stylus when it was located at the highest and lowest positions in the FOV of the camera, respectively. The calibration process was based on determining the diameter and spherical center of the ruby ball from its captured images. To this end, a composite edge detection and distance measuring function within the $\mathrm{NI}$ LabView/Vision Assistant, the clamp function [4], was applied first to roughly determine the upper and lower boundary edge points of the ruby ball, $B_{1}$ and $B_{2}$, with an appropriate rectangular region of interest (called ROI 1), specified as shown in Figure 7(a). Edge points within ROI 1 can be detected by finding sudden changes of grayscale intensity values along the vertical direction. A circular edge detection function within the $\mathrm{NI}$ LabView/Vision Assistant, the spoke function [4], was then applied to precisely determine the diameter and spherical center of the ruby ball, $D_{C}$ and $P_{C}$, through a least squares circle-fitting approach using the detected edge points along the radial direction, as shown in Figure $7(b)$. An open annular ROI (called ROI 2) is specified for executing the spoke function; the annular center is specified as the midpoint of the points $B_{1}$ and $B_{2}$. The individual ruby ball diameters $D_{C}$ in all captured images were determined and averaged to obtain an average ball diameter of 439.351 pixels. Thus, the conversion factor of the captured digital image corresponding to its real world dimensions was found to be $2.276 \mu \mathrm{m} /$ pixel (= $1 \mathrm{~mm} /$ 439.351 pixels). By applying the sub-pixel localization algorithm $[4,5]$, the machine vision module was able to achieve an estimated resolution of $1 / 25$ pixel [4]: i.e., $0.071 \mu \mathrm{m}$. In addition, after the spherical centers $P_{C}$ of the ruby ball in all captured images were located, their vertical components, $P_{C y}$, could be formulated for each image as

$$
P_{c y}(s)=e \sin (\theta(s))+P_{y 0}=e \sin \left(s+s_{0}\right)+P_{y 0}
$$

where $e$ is the eccentricity, $\theta$ is the phase angle, $P_{y 0}$ is the vertical location of the rotational axis, $s$ is the angular position of the motor (according to step counts), and $s_{0}$ is a constant phase difference between $\theta$ and $s$. With this definition, $s=0^{\circ}$ corresponds to the stylus being at an arbitrary initial angular position. When $\theta=90^{\circ}$, the stylus would be located at its highest position, and when $\theta=$ $270^{\circ}$, it would be at its lowest position. Since the vertical height $P_{c y}(s)$ can be found through image processing, $e$, $P_{y 0}$, and $s_{0}$ can all be determined by a least squares sine-fitting approach [6]. Accordingly, the function $\theta(s)$ can be determined. Figure 7 (c) shows a calibration result for the angular phase. In this case, values of $e=0.355$ $\mathrm{mm}$ ( $=156.053$ pixels), $P_{y 0}=1106.258$ pixels (relative to the bottom of the FOV) and $s_{0}=80.947^{\circ}$ were obtained: thus, $\theta=s+80.947^{\circ}$. After performing the calibration process, the insert would then be positioned at $\theta=90^{\circ}$ (as in Figure 4(a)) and $\theta=0^{\circ}$ (as in Figure $4(\mathrm{~b})$ ) for inspection tasks.

\section{The Defect Detection}

The defect detection aims to determine whether the sintered blanks of the inserts have shape defects that would constitute excessive volumetric losses. Such volumetric losses inevitably influence the shape correctness of form-ground semi-products. In standard PM practice, after performing compaction and before 
sintering, the tool tip and edge corner portions of the blanks (also called green compacts at that state) are most likely to break during die release, and therefore detection of any resultant shape defects is necessary.

For defect detection, side and face images of sintered blanks were captured and processed for indirectly evaluating the volumetric losses of the blanks. The captured side image in Figure $8(a)$ is of a qualified sintered blank, and that in Figure $8(\mathrm{~b})$ is of an unqualified one. The upper-left tool tip portion of the unqualified blank was evidently broken during die release. A simple and practical method for indirectly evaluating the volumetric loss is to calculate its corresponding projected area loss. Figure 9 shows a solution for the side image case: by specifying an appropriate rectangular ROI (called ROI 3) covering the tool tip portion, a triangular area $A_{S(i)}$ representing the ideal tip shape can be determined by finding two straight edges $I_{1}$ and $I_{2}$ (with the use of edge detection [4, 5] and the linear least squares approach [7]) and their intersection $Q$ to calculate the area of triangle $\mathrm{QB}_{3} \mathrm{~B}_{4}$. The actual area of the tool tip portion $A_{S(a)}$ (the cyan region shown in Figure 9 ) is then calculated by using thresholding [4] and dark pixel counts. The area ratio $A_{S(a)} / A_{S(i)}$ can be obtained to indirectly evaluate the volumetric loss. In practice, a criterion of $A_{S(a)} / A_{S(i)}>0.95$ could be given in judging whether a sintered blank is qualified or unqualified. For the sintered blank shown in Figure $8(a)$, the area ratio was 0.995 , and that of the one shown in Figure 8(b) was 0.829. The latter was obviously unqualified.

Figure 10(a) shows the captured face image of a qualified sintered blank, and Figure 10(b) shows that of an unqualified one. As observed, the lower-left edge corner portion of the unqualified blank was broken during die release. Figure 11 shows the solution for the face image case: two appropriate rectangular ROls (called ROI 4 and ROI 5) are specified to cover the upper-left and lower-left edge corner portions; two quadrangle areas $A_{F 1(i)}$ and $A_{F 2(i)}$ representing their ideal edge corner shapes can then be determined in a similar manner to the side image case. The actual areas of the edge corner portions $A_{F 1(a)}$ and $A_{F 2(a)}$ (the upper and lower cyan areas shown in Figure 11) are calculated by using thresholding [4] and dark pixel counts. Two area ratios, $A_{F 1(a)} / A_{F 1(i)}$ and $A_{F 2(a)} / A_{F 2(i)}$, can be obtained to evaluate the volumetric losses indirectly. In practice, criteria of $A_{F 1(a)} / A_{F 1(i)}>0.95$ and $A_{F 2(a)} / A_{F 2(i)}>0.95$ for judging qualified sintered blanks could be given. For the sintered blank shown in Figure 10(a), its upper and lower area ratios were 0.976 and 0.985 , respectively, and those of the one shown in Figure 10 (b) were 0.984 and 0.925 , respectively; thus, the lower edge corner of the latter was deemed unqualified.

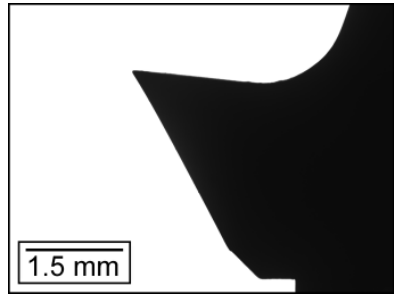

(a)

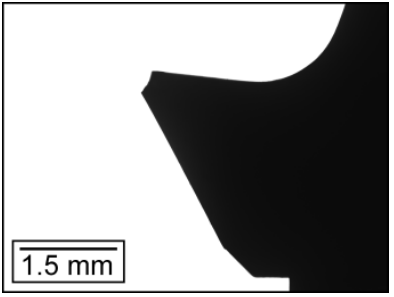

(b)
Figure 8. Captured side images of two sintered blanks: (a) a qualified one and (b) an unqualified one.

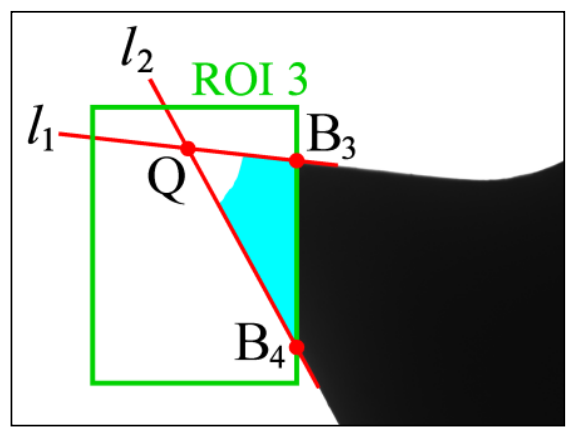

Figure 9. Illustrated example of defect detection for the side image of a sintered blank.

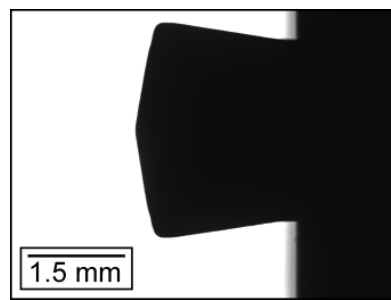

(a)

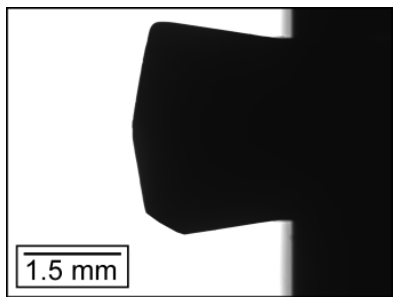

(b)
Figure 10. Captured face images of two sintered blanks: (a) a qualified one and (b) an unqualified one.

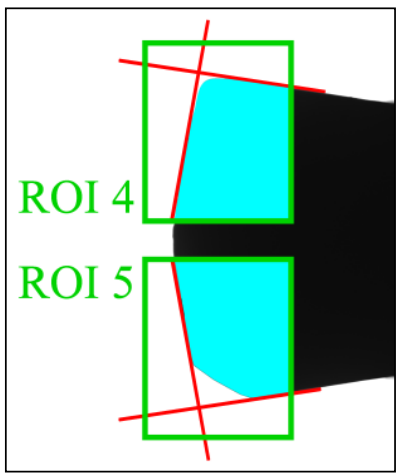

Figure 11. Illustrated example of defect detection for the face image of a sintered blank.

\section{The Geometric Measurement}

The geometric measurement aims to inspect the geometric accuracy of form-ground semi-products of the inserts, as their accuracy influences sawing performance. 


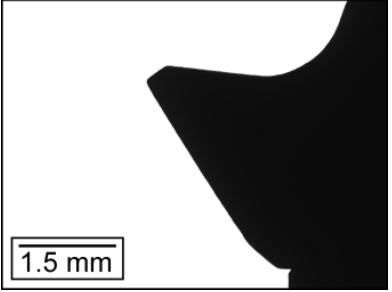

(a)

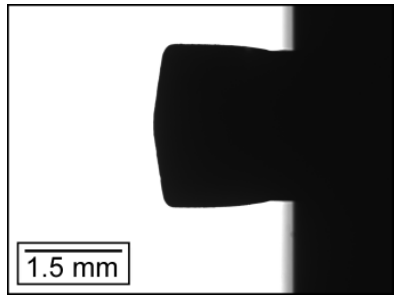

(b)
Figure 12. Captured images of a form-ground insert: (a) the side image and (b) the face image.

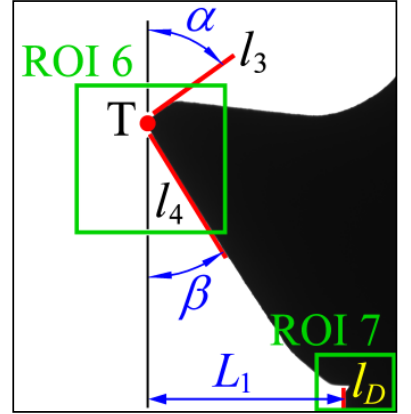

(a)

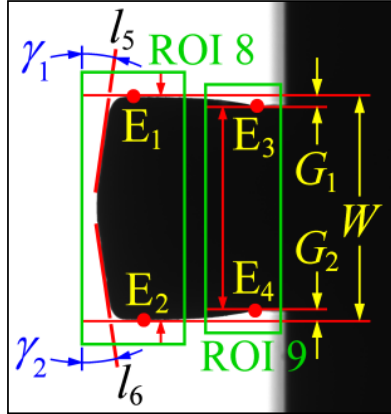

(b)
Figure 13. Illustrated examples of the geometric measurement of a form-ground insert: (a) results of the side image and (b) results of the face image.

In the geometric measurement process, side and face images of form-ground inserts were captured, and these images processed for measuring their projective dimensions. Figure 12(a) shows the captured side image of a form-ground insert, and its captured face image is shown in Figure 12(b).

From the side image, the overall length $L$, face angle $\alpha$ and flank angle $\beta$ of the insert (as shown in Figure 2) can be measured. As shown in Figure 13(a), by specifying two appropriate rectangular ROls (called ROI 6 and ROI 7) to cover the tool tip portion and the front datum of the lower member, respectively, the tool tip $T$ and the straight edge of the front datum $I_{D}$ can be found (with the use of edge detection [4, 5] and the linear least squares approach [7]). The horizontal distance, $L_{1}$ between point $T$ and line $I_{D}$, can then be obtained. The overall length $L$ can be evaluated by

$$
L=L_{0}+L_{1}
$$

where $L_{0}$ is the perpendicular distance between the front and back datums that had been measured. Then, in ROI 6, two straight edges $I_{3}$ and $I_{4}$ representing the projective face and flank edges of the insert, respectively, can be obtained with the use of edge detection $[4,5]$ and the linear least squares approach [7]. The face angle $\alpha$ can thus be evaluated by calculating the inclined angle between line $I_{3}$ and the vertical direction; the flank angle $\beta$ can be evaluated similarly, by calculating the inclined angle between line $I_{4}$ and the vertical direction.
Likewise, from the face image, the overall width $W$, upper gap $G_{1}$, lower gap $G_{2}$, upper edge angle $\gamma_{1}$ and lower edge angle $\gamma_{2}$ of the insert (as shown in Figure 2) can be measured. As shown in Figure 13(b), by applying the clamp function [4] with two appropriate ROls (called ROI 8 and ROI 9) specified, the upper and lower extreme edge points within the two ROls, $E_{1}, E_{2}, E_{3}$ and $E_{4}$ can be determined. The overall width $W$ of the insert can be evaluated by calculating the vertical distance between points $E_{1}$ and $E_{2}$, the upper gap $G_{1}$ by calculating the vertical distance between points $E_{1}$ and $E_{3}$, and the lower gap $G_{2}$ by calculating the vertical distance between points $\mathrm{E}_{2}$ and $\mathrm{E}_{4}$. Additionally, in ROI 8, two straight edges $I_{5}$ and $I_{6}$, representing the respective projected upper and lower cutting edges of the insert, can be obtained with the use of edge detection [4, 5] and the linear least squares approach [7]. The upper or lower edge angle, $\gamma_{1}$ or $\gamma_{2}$, can then be evaluated by calculating the inclined angle between line $I_{5}$ and the vertical direction or between line $I_{6}$ and the vertical direction, respectively.

Table 1. Experimental results of defect detection.

\begin{tabular}{llll}
\hline \hline Area ratio & Sample SB1 & Sample SB2 & Sample SB3 \\
\hline$A_{S(a)} / A_{S(i)}$ & $0.993 \pm 0.006$ & $\mathbf{0 . 8 3 0} \pm \mathbf{0 . 0 1 1}$ & $0.988 \pm 0.008$ \\
$A_{F 1(a)} / A_{F 1(i)}$ & $0.977 \pm 0.008$ & $0.980 \pm 0.017$ & $0.985 \pm 0.013$ \\
$A_{F 2(a)} / A_{F 2(i)}$ & $0.984 \pm 0.014$ & $0.982 \pm 0.009$ & $\mathbf{0 . 9 2 3} \pm \mathbf{0 . 0 0 7}$ \\
\hline \hline
\end{tabular}

\section{Preliminary Experimental Results}

Two experiments were conducted to inspect some insert samples and to test the feasibility of the developed prototype $\mathrm{AOI}$ system and image processing procedures.

In the first experiment, three sintered blanks (called samples SB1, SB2, and SB3) were each held by the gripper and their side and face images captured once, then immediately removed from the gripper. This operation was repeated thirty times. The developed defect detection method was applied to evaluate their volumetric losses. Table 1 shows the results of this experiment, in which the area ratios are presented as averaged values with uncertainties evaluated by the well-known three-standard-deviation-band approach [8]. Evidently, some of the area ratios of samples SB2 and SB3 were less than 0.95; both samples were thus considered unqualified. In addition, the largest uncertainty for this experiment was within the range of \pm 0.02 (i.e., $\pm 2 \%$ ). As a result, the developed defect detection method was preliminarily verified as feasible for inspecting certain sintered blanks. 
Table 2. Experimental results of geometric measurement.

\begin{tabular}{llllll}
\hline \hline Parameter & Unit & Qualified range & Sample FG1 & Sample FG2 & Sample FG3 \\
\hline Overall length $L$ & $\mathrm{~mm}$ & $11.89 \sim 11.95$ & $11.926 \pm 0.005$ & $11.935 \pm 0.004$ & $11.920 \pm 0.004$ \\
Overall width $W$ & $\mathrm{~mm}$ & $2.47 \sim 2.53$ & $\mathbf{2 . 5 3 9} \pm \mathbf{0 . 0 0 2}$ & $\mathbf{2 . 5 2 5 \pm 0 . 0 0 1}$ & $2.515 \pm 0.002$ \\
Upper gap $G_{1}$ & $\mathrm{~mm}$ & $0.095 \sim 0.115$ & $0.114 \pm 0.001$ & $\mathbf{0 . 1 2 0} \pm \mathbf{0 . 0 0 1}$ & $0.113 \pm 0.000$ \\
Lower gap $G_{2}$ & $\mathrm{~mm}$ & $0.095 \sim 0.115$ & $0.110 \pm 0.002$ & $\mathbf{0 . 0 9 2} \pm \mathbf{0 . 0 0 1}$ & $0.111 \pm 0.001$ \\
Face angle $\alpha$ & degree & $53.0 \sim 54.0$ & $53.526 \pm 0.157$ & $\mathbf{5 4 . 1 4 1} \pm \mathbf{0 . 1 3 2}$ & $53.497 \pm 0.127$ \\
Flank angle $\beta$ & degree & $31.0 \sim 32.0$ & $31.817 \pm 0.147$ & $31.342 \pm 0.145$ & $31.682 \pm 0.153$ \\
Upper edge angle $\gamma_{1}$ & degree & $7.9 \sim 8.5$ & $\mathbf{8 . 8 5 2} \pm \mathbf{0 . 1 2 6}$ & $\mathbf{8 . 8 1 2} \pm \mathbf{0 . 0 3 2}$ & $8.303 \pm 0.064$ \\
Lower edge angle $\gamma_{2}$ & degree & $7.9 \sim 8.5$ & $8.043 \pm 0.076$ & $8.404 \pm 0.061$ & $8.334 \pm 0.036$ \\
\hline \hline
\end{tabular}

In the second experiment, three form-ground inserts (called samples FG1, FG2, and FG3) were each held by the gripper and their side and face images captured once, then immediately removed from the gripper. This operation was repeated thirty times. The developed geometric measurement method was applied to inspect their geometric accuracy. Table 2 shows the results of this experiment presented as averaged values with uncertainties. The measured overall width $W$ and upper edge angle $\gamma_{1}$ of sample FG1 were outside their qualified ranges. Also, the measured upper gap $G_{1}$, lower gap $G_{2}$, face angle $\alpha$ and upper edge angle $\gamma_{1}$ of sample FG2 were outside their qualified ranges. The measured values of sample FG3 were all qualified. In addition, the uncertainty of this experiment was within the range of $\pm 5 \mu \mathrm{m}$ or $\pm 0.16^{\circ}$. Therefore, the developed geometric measurement method was preliminarily verified as feasible for inspecting certain form-ground inserts.

\section{Discussion}

The preliminary experimental results show that the presented prototype $\mathrm{AOI}$ system and image processing procedures were feasible for inspecting certain circular saw inserts for quality control. In other words, the developed AOI system could be integrated with numerically controlled (NC) grinding machines, conveyor systems, and other related automation components to accomplish an automated production line of circular saw inserts. Nevertheless, the gripper used in the developed AOI system would require some modifications for it to be manipulated automatically. If this can be achieved, mass production of circular saw inserts could be carried out almost entirely by computer-aided automated processes. In addition, the concepts of the presented system and method could also be applied to inspect other types of cutting tool inserts if some necessary modifications are made. Furthermore, general-purpose production systems for cutting tool inserts could be developed based on integration of the presented $\mathrm{AOI}$ system, NC machining tools, automation processes, and intelligent software.

\section{Conclusion}

The preliminary development of an AOI system for quality control of Yih Troun circular saw inserts has been introduced in this paper. A prototype inspection system mainly consisting of a rotary gripper module and a machine vision module was designed and constructed. Image processing procedures were also developed to calibrate the prototype system, detect defects in the sintered blanks, and measure the geometry of the form-ground semi-products. From preliminary experimental results, the uncertainty of the defect detection method was seen to not exceed $\pm 2 \%$, and those of the geometric measurement method to not exceed $\pm 5 \mu \mathrm{m}$ or $\pm 0.16^{\circ}$. Therefore, the presented prototype AOI system and image processing procedures were preliminarily verified as feasible for the inspection of certain circular saw inserts for quality control. Integrating the prototype inspection system into an automated production line of circular saw inserts could be one possible direction for future work on this topic.

\section{Acknowledgement}

The authors are grateful to Yih Troun Enterprise Corporation for providing samples of circular saw inserts for the experimental work.

\section{References}

[1] W. T. Chang, C. H. Su, D. X. Guo, G. R. Tang, and F. J. Shiou, "Automated optical inspection for the runout tolerance of circular saw blades," The International Journal of Advanced Manufacturing Technology, pp. 1-18, 2012. doi: $10.1007 / \mathrm{s} 00170-012-4350-6$

[2] E. P. DeGarmo, J. T. Black, and R. A. Kohser, Materials and processes in manufacturing, 9 ed. New Jersey: Wiley, 2002. 
[3] Indexable saw blade, Yih Troun Enterprise Corporation, [Online].

Available:

http://www.cut-tools.com.tw/index en.php

[4] NI vision concepts manual. Austin, TX: National Instrument Corp., 2007.

Available:

http://digital.ni.com/manuals.nsf/websearch/80CC 7F2386F7701F8625731500701D23
[5] R. Jain, R. Kasturi, and B. G. Schunck, Machine vision. New York: McGraw-Hill, 1995.

[6] G. A. F. Seber and C. J. Wild, Nonlinear regression. Hoboken, NJ: Wiley, 2003.

[7] J. D. Faires and R. L. Burden, Numerical methods, 3 ed. Belmont, CA: Thomson Brooks/Cole, 2003.

[8] T. G. Beckwith, R. D. Marangoni, and J. H. Lienhard, Mechanical measurements, 5 ed. Taipei: Pearson Education Taiwan, 2004. 